\title{
The Role of Proton Mobility in Determining the Energy-Resolved Vibrational Activation / Dissociation Channels of N-Glycopeptide Ions
}

\author{
Venkata Kolli, ${ }^{1}$ Heidi A. Roth, ${ }^{1}$ Gabriela De La Cruz,,${ }^{1,2}$ \\ Ganga S. Fernando, ${ }^{1,2}$ and Eric D. Dodds ${ }^{1 *}$ \\ ${ }^{1}$ Department of Chemistry, University of Nebraska - Lincoln \\ Lincoln, NE, 68588-0304, USA \\ ${ }^{2}$ Department of Chemistry, Cottey College \\ Nevada, MO, 64772, USA \\ *Corresponding Author \\ Department of Chemistry, University of Nebraska - Lincoln \\ 711 Hamilton Hall, Lincoln, NE, 68588-0304 \\ E-mail edodds2@unl.edu; Telephone 1-402-472-3592; Fax 1-402-472-9402 \\ Submitted to Analytica Chimica Acta \\ For Consideration as a Full Length Article \\ 23 June 2015 \\ Submitted in Revised Form: \\ 04 September 2015 \\ (C) 2016. This manuscript version is made available under the Elsevier user license \\ http://www.elsevier.com/open-access/userlicense/1.0/
}




\section{Abstract}

Site-specific glycoproteomic analysis largely hinges on the use of tandem mass spectrometry (MS/MS) to identify glycopeptides. Experiments of this type are usually aimed at drawing connections between individual oligosaccharide structures and their specific sites of attachment to the polypeptide chain. These determinations inherently require ion dissociation methods capable of interrogating both the monosaccharide and amino acid connectivity of the glycopeptide. Collision-induced dissociation (CID) shows potential to satisfy this requirement, as the vibrational activation / dissociation of protonated $\mathrm{N}$ glycopeptides has been observed to access cleavage of either glycosidic bonds of the glycan or amide bonds of the peptide in an energy-resolved manner. Nevertheless, the relative energy requirement for these fragmentation pathways varies considerably among analytes. This research addresses the influence of proton mobility on the vibrational energy necessary to achieve either glycan or peptide cleavage in a collection of protonated $\mathrm{N}$-glycopeptide ions. While greater proton mobility of the precursor ion was found to correlate with lower energy requirements for precursor ion depletion and appearance of glycosidic fragments, the vibrational energy deposition necessary for appearance of peptide backbone fragments showed no relation to the precursor ion proton mobility. These results are consistent with observations suggesting that peptide fragments arise from an intermediate fragment which is generally of lower proton mobility than the precursor ion. Such findings have potential to facilitate the rational selection of CID conditions which are best suited to provide either glycan or peptide cleavage products in MS/MS based N-glycoproteomic analysis.

\section{Keywords}

Glycoproteins; glycopeptides; glycoproteomics; tandem mass spectrometry; collision-induced dissociation; proton mobility. 


\section{Introduction}

Mass spectrometry (MS) based glycoproteomics is an emerging branch of postgenomic analytical science that lies at the intersection of proteomics and glycomics [1-4]. While glycoproteomic experiments are conducted with widely varied scopes and objectives, they are generally aimed at the identification or characterization of glycosylated proteins; the compositional and / or structural determination of protein-linked glycans; and the association of individual glycan compositions and / or structures with specific sites of attachment to the protein [5-8]. Accordingly, a wide assortment of MS centered tools are actively applied to glycoproteomics [9-12]. In many of these approaches, tandem mass spectrometry (MS/MS) of glycosylated proteolytic fragments plays a critical role, often serving as the final analytical readout of the experiment $[13,14]$.

Although MS/MS analysis of glycopeptides is analytically demanding (polypeptide sequence and oligosaccharide connectivity are simultaneously involved), these experiments can provide a level of molecular detail not afforded by methods which release the glycan from the protein prior to analysis $[15,16]$. Nevertheless, complete elucidation of glycopeptide topology requires MS/MS methods capable of providing information on both the amino acid sequence and the monosaccharide connectivity. While this is frequently accomplished by combining multiple, complementary ion fragmentation methods [13, 14], several such methods have been shown capable of probing both the oligosaccharide and polypeptide moieties of glycopeptides. These include ultraviolet photodissociation (UVPD) $[17,18]$, infrared multiphoton dissociation (IRMPD) [19-22], and low-energy beam-type collision-induced dissociation (CID) [23-26].

By a wide margin, CID is the most widely available ion dissociation method for MS/MS. While a disadvantage of CID is loss of the glycan prior to the onset of peptide fragmentation, in most cases this does not hinder the localization of N-glycosylation, which only occurs within the context of a consensus sequon (multiple instances of which rarely appear on the same proteolytic fragment). Therefore, extending the applicability of CID for 
$\mathrm{N}$-glycopeptide analysis is of significant interest. One barrier towards an expanded role for CID in N-glycoproteomics is that the distinct energetic requirements for accessing glycosidic bond cleavages or peptide backbone cleavages are not well understood in terms of the physicochemical characteristics of the precursor ions $[27,28]$.

The present work is focused on the relationship between precursor ion proton mobility [29, 30] and optimum collision energies for accessing glycan versus peptide scission. Energy-resolved CID studies were conducted on a group of protonated Nglycopeptides in which different amino acid compositions and charge states were represented. In general, precursor ion proton mobility correlated negatively with the collision energies necessary to deplete the precursor ion and provide glycosidic cleavage; however, the collision energies needed to achieve peptide fragmentation had no noticeable relationship to the proton mobility of the precursor ion. This is consistent with energyresolved CID results that show the peptide fragmentation products arise from an intermediate fragment of low proton mobility. On the whole, these findings suggest that, with adequate knowledge of the relevant fragmentation processes, CID could be conducted such that the monosaccharide connectivity and amino acid sequence of protonated $\mathrm{N}$ glycopeptides could be deliberately accessed.

\section{Experimental}

2.1. Reagents and materials. Ammonium bicarbonate, bovine ribonuclease $B$ (BRB), dithiothreitol, formic acid, imidazole, iodoacetamide, proteomics grade trypsin, and urea were all procured from Sigma-Aldrich (St. Louis, MO, USA). Acetonitrile (HPLC grade) and water (HPLC grade) were obtained from Fisher Scientific (Fair Lawn, NJ, USA) and Burdick \& Jackson (Muskegon, MI, USA), respectively. Zwitterionic hydrophilic interaction liquid chromatography (ZIC-HILIC) micropipette tips for solid phase extraction (SPE) were acquired from Protea Biosciences (Somerset, NJ, USA). 
2.2. Glycopeptide preparation. A $50 \mu \mathrm{L}$ aliquot of $2 \mu \mathrm{g} \mu \mathrm{L}^{-1}$ BRB glycoprotein solution in $8 \mathrm{M}$ urea and $50 \mathrm{mM} \mathrm{NH}_{4} \mathrm{HCO}_{3}(\mathrm{pH}$ 7.5) was subjected to disulfide bond reduction by addition of $10 \mu \mathrm{L}$ of $450 \mathrm{mM}$ dithiothreitol in $50 \mathrm{mM} \mathrm{NH}{ }_{4} \mathrm{HCO}_{3}(\mathrm{pH} 7.5$; incubated $1 \mathrm{~h}$ at $55^{\circ} \mathrm{C}$ ). The sample was next subjected to thiol alkylation by treatment with $10 \mu \mathrm{L} 500 \mathrm{mM}$ iodoacetamide in $50 \mathrm{mM} \mathrm{NH}_{4} \mathrm{HCO}_{3}(\mathrm{pH} \mathrm{7.5}$; incubated $1 \mathrm{~h}$ at room temperature and in the

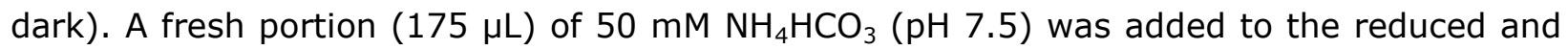
alkylated glycoprotein sample such that the total urea concentration was reduced to $<2 \mathrm{M}$. Proteolysis was then performed through addition of a $5 \mu \mathrm{L}$ aliquot of $0.5 \mu \mathrm{g} \mu \mathrm{L}^{-1}$ of trypsin (incubated $18 \mathrm{~h}$ at $37^{\circ} \mathrm{C}$ ). The resulting tryptic digest was reduced to $\sim 10 \mu \mathrm{L}$ in volume via vacuum centrifugation (Speed Vac SC110, Thermo Savant, Holbrook, NY, USA), then reconstituted in $0.1 \% \mathrm{HCOOH}$ to a final volume of $\sim 100 \mu \mathrm{L}$. Glycopeptides from an aliquot of this solution ( $4 \mu \mathrm{L}$ reconstituted digest diluted to a total volume of $20 \mu \mathrm{L}$ with $\mathrm{CH}_{3} \mathrm{CN}$ ) were enriched and purified using ZIC-HILIC SPE. Briefly, the SPE micropipette tip was equilibrated with $80 \% \mathrm{CH}_{3} \mathrm{CN} / 0.1 \% \mathrm{HCOOH}$, loaded with the sample aliquot described above, washed with $80 \% \mathrm{CH}_{3} \mathrm{CN} / 0.1 \% \mathrm{HCOOH}$, and finally eluted in $20 \mu \mathrm{L} 0.1 \% \mathrm{HCOOH}$. In some cases, purified digests were spiked to contain $10 \mathrm{mM}$ imidazole. This was done in order to enhance the production of glycopeptide ions with lower charge states for study.

2.3. Mass spectrometry and data analysis. The purified glycopeptide digest was prepared for MS analysis by placing a portion of ZIC-HILIC SPE eluate $(\sim 5-10 \mu \mathrm{L})$ into a home-pulled glass emitter for nanoelectrospray ionization (nESI). This transfer was accomplished using a Hamilton $10 \mu \mathrm{L}$ taper-tipped syringe (Reno, NV, USA). The nESI emitters were prepared from $1.5-1.8$ x 100 mm melting point capillary tubes (Corning Pyrex, Corning, NY, USA) with the aid of a vertical micropipette puller (David Kopf Instruments, Tujunga, CA, USA). The filled emitter was then fitted to a custom-built holder which made use of a platinum wire to provide the nESI potential directly to the analyte solution. This apparatus was then adapted onto the commercial nESI source of a Waters Synapt G2 HDMS quadrupole time of flight (Q-TOF) hybrid mass spectrometer (Manchester, 
UK). Ion source conditions for nESI included an emitter potential of $1.0-1.4 \mathrm{kV}$, a sampling cone potential of $15-35 \mathrm{~V}$, an extraction cone potential of $2-4 \mathrm{~V}$, and a temperature of $80^{\circ} \mathrm{C}$. For MS/MS experiments, quadrupole selection of precursor ions was followed by CID in the "trap" region of the instrument (a stacked ring ion guide containing argon at a pressure of approximately $5.0 \times 10^{-3}$ mbar). The DC offset $(\Delta U)$ which determined the kinetic energy of ions entering the collision cell was systematically adjusted in order to obtain energy-resolved CID spectra.

All instrument control and data acquisition was conducted with the use of MassLynx 4.1 (Waters). Subsequent data handling and graph generation was performed using IGOR Pro 6.3 (WaveMetrics, Lake Oswego, OR, USA) and SigmaPlot 10.1 (Systat, Chicago, IL, USA). For schematic purposes, standard one-letter amino acid abbreviations were used in diagramming the peptide moiety, while glycan structures were diagrammed using the conventions suggested by Varki et al. [31]. The assignment of fragment ions was performed in accord with the formalisms of Domon and Costello (for glycan fragments) and Roepstorff and FohIman (for peptide fragments) [32, 33]. In accord with these systems of nomenclature, lower case letters were used to indicate peptide fragments, while upper case letters were used to indicate glycan fragments. If multiple glycan cleavages (or combinations thereof) could plausibly yield a given product ion mass, the fragment ion was assigned by indicating the composition of losses from the precursor ion (e.g., $\left[\mathrm{M}-\mathrm{Man}_{2}\right]^{2+}$ ). For simplicity, small neutral losses from precursor or fragment ions (e.g., $\mathrm{NH}_{3}, \mathrm{H}_{2} \mathrm{O}$ ) were not labeled in the spectra. Monosaccharide names were abbreviated as follows: GlcNAc, Nacetylglucosamine; Man, mannose.

\section{Results}

3.1. Overview. As described above, a set of model glycopeptide analytes was prepared by trypsinolysis of BRB with the goal of quantitatively characterizing the influence of proton mobility upon the energy-resolved vibrational activation / dissociation pathways of 
selected $\mathrm{N}$-glycopeptides. The presence of several potential tryptic cleavage sites in close proximity to the single glycosylation site of $B R B$, coupled with the tendency of glycosylation to sterically interfere with protease action [34-37], resulted in the production of glycopeptides with multiple amino acid sequences based on a combination of the fully tryptic cleavage product and various partially tryptic cleavage products. Specifically, Nglycopeptides with the amino acid sequences NLTK, NLTKDR, SRNLTK, and SRNLTKDR were obtained. This provided a group of model glycopeptide analytes which encompassed some key variations in sequence, particularly with respect to the number and location of basic sites. Protonated glycopeptide ions based on the above peptide groups carrying the GlcNAc $_{2} \mathrm{Man}_{5}$ high mannose $\mathrm{N}$-glycan were each studied in two charge states by energyresolved CID, as described in detail below.

3.2. Precursor ion survival curves. MS/MS data were collected via CID for each of eight glycopeptide precursor ions: NLTK $+\mathrm{GlcNAc}_{2} \mathrm{Man}_{5}$ as the $[\mathrm{M}+\mathrm{H}]^{+}$and $[\mathrm{M}+2 \mathrm{H}]^{2+}$ ions; $\mathrm{NLTKDR}+\mathrm{GlcNAc}_{2} \mathrm{Man}_{5}$ as the $[\mathrm{M}+2 \mathrm{H}]^{2+}$ and $[\mathrm{M}+3 \mathrm{H}]^{3+}$ ions; SRNLTK + $\mathrm{GlcNAC}_{2} \mathrm{Man}_{5}$ as the $[\mathrm{M}+2 \mathrm{H}]^{2+}$ and $[\mathrm{M}+3 \mathrm{H}]^{3+}$ ions; and SRNLTKDR $+\mathrm{GICNAC}_{2} \mathrm{Man}_{5}$ as the $[\mathrm{M}+2 \mathrm{H}]^{2+}$ and $[\mathrm{M}+3 \mathrm{H}]^{3+}$ ions. In these experiments, CID was conducted at various applied $\Delta U$ values, which were adjusted in $2.5-10.0 \mathrm{~V}$ increments, depending on the analyte. The resulting spectra were used to prepare precursor ion survival curves by plotting the percent fractional area of the precursor ion peak as a function of the applied collision energy. As depicted in Figure 1, this relatively small set of related glycopeptide precursor ions (i.e., all bearing the same glycan composition, and all derived from the same glycosylation site) exhibited a remarkably broad range of energy-dependent stabilities, with the applied $\Delta U$ resulting in < $20 \%$ precursor ion survival ranging from approximately $10.0 \mathrm{~V}-60.0 \mathrm{~V}$. This underscores a key challenge in the interrogation of glycopeptide connectivity by CID; namely, the selection of collision energies yielding CID spectra which are most informative of the overall topology of a given glycopeptide precursor ion. This is particularly important given the ability of vibrational activation / dissociation methods to provide fragments which can be informative 
of either the oligosaccharide connectivity or the polypeptide sequence, depending on the amount of vibrational energy deposited $[25,26]$.

While Figure 1 is useful in illustrating the highly varied degrees of precursor ion depletion as a function of $\Delta U$ (i.e., the instrumental parameter which is most directly used to determine the amount of vibrational energy deposited into the precursor ion), comparisons between the stabilities of the various precursor ions are difficult to rationalize from visual inspection of these data alone. Indeed, the precursor ion survival behaviors are dictated by not only the compositions of the analyte ions, but also their charge states and number of vibrational degrees of freedom $[38,39]$. To allow more meaningful comparisons among the various analytes studied, the initial kinetic energies which resulted in $50 \%$ depletion of the precursor were calculated and normalized for the number of vibrational modes available to the analyte. This was carried out by first applying a linear fit to the steepest region of each precursor ion survival curve, then determining value of $\Delta U$ which corresponded to $50 \%$ fractional intensity of the precursor ion. These values were in turn used to calculate the corresponding initial kinetic energy, $E_{k}$ (expressed with units of eV), of the precursor ion:

$$
E_{k}=z \Delta U
$$

Equation 1

Here, $z$ represents the precursor ion charge state expressed as an integer multiple of the fundamental charge. These values, which account for the charge state of the precursor ion, were further normalized for the number of vibrational degrees of freedom, $f_{v}$, available to the precursor ion. For an analyte composed of $n$ atoms:

$$
f_{v}=3 n-6
$$

Equation 2

The vibrational degrees of freedom normalized initial precursor ion kinetic energy, $E_{k, n}$, was then defined and calculated according to:

$$
E_{k, n}=\frac{10^{2} E_{k}}{f_{v}}
$$


where the factor of $10^{2}$ was applied to provide more convenient values for comparison (thus, the values of $E_{k, n}$ are $10^{2}$ times the number of electron volts per vibrational mode). The values of $E_{k, n}$ corresponding to the $50 \%$ survivals of all precursor ions studied are provided in Table 1. In general, the vibrational energy deposition required to deplete $50 \%$ of the initial precursor ion population was found to negatively correlate with proton mobility, as estimated based on the relative number of charge-carrying protons $\left(n_{H}\right)$ and basic amino acid residues $\left(n_{\mathrm{B}}\right)$ present. For each precursor ion studied here, the charge-carrying proton(s) could be described as "mobile," "partially mobile," or "nonmobile." These categories, suggested by Kapp et al., were based on empirically determined "cleavage intensity ratios" (CIRs) which were measured according to the relative proportions of fragmentation products known to arise from processes that occur preferentially in the absence of mobile protons [40]. For instance, peptide cleavages C-terminal to aspartic acid residues occur preferentially in precursor ions with low proton mobility [41, 42]. Therefore, the proportion of this type of fragment relative to the total fragment population can be indicative of the extent of proton mobility. Accordingly, CIR values increase with decreasing proton mobility. In the present experiments, glycopeptide precursor ions with $n_{\mathrm{H}}>n_{\mathrm{B}}$, which thus contained a readily mobile proton (with CIRs ranging from 0.73 - 0.90), exhibited $50 \%$ precursor ion survivals at $E_{k, n}$ values ranging from $1.80-4.18$. For glycopeptide ions with $n_{\mathrm{H}}=n_{\mathrm{B}}$, thus rendering the protons partially mobile (with CIRs ranging from $1.52-2.18$ ), the $E_{k, n}$ values corresponding to $50 \%$ precursor ion survival were significantly higher on average, ranging from 4.97 - 7.57. Finally, one analyte in which all protons were considered nonmobile (CIR of 4.58) had a $50 \%$ precursor ion survival occurring at an $E_{k, n}$ value of 9.14. Taken together, these data demonstrate that the order of charge state corrected and vibrational degrees of freedom normalized initial precursor ion kinetic energies which brought about $50 \%$ precursor ion depletion can be rationalized by proton mobility; that is, those precursor ions characterized by lower proton mobility required greater vibrational energy deposition per vibrational mode in order to bring about 
unimolecular dissociation processes. Nevertheless, the normalized collision energies needed to access an analytically useful degree of precursor ion fragmentation varied substantially within this group of glycopeptides which, in many ways, would be considered quite similar (all derived from the same glycosylation site, and all bearing the same glycan).

3.3. Energy-resolved CID comparisons at various proton mobilities. In order to further delineate the role of proton mobility in the vibrational activation / dissociation behavior of glycopeptides, energy-resolved breakdown curves were plotted for each precursor ion under study. As discussed in section 2.3 above, all glycan and peptide fragment ion designations have been applied according to accepted systems of nomenclature $[32,33]$. At each collision energy, the total peak areas were determined for each of several product ion categories: the precursor ion, $[\mathrm{M}+n \mathrm{H}]^{n+}$; the larger $Y$-type glycosidic bond cleavage products, $\Sigma Y_{n>1}$; the peptide chain with one remaining GlcNAc residue attached, $Y_{1}$; the bare peptide chain, $Y_{0}$; cross-ring cleavage of the reducing terminal GlcNAc residue, ${ }^{0,2} X_{0}$; and peptide backbone fragmentation products, $\Sigma(b+y)$. The fraction of the total spectral peak area arising from fragment ions in each of these categories were then plotted against collision energy, expressed as the applied $\Delta U$. In Figure 2, CID breakdown curves are presented for three representative glycopeptide ions with differing proton mobilities: $\left[\mathrm{NLTKDR}+\mathrm{GlcNAC}_{2} \mathrm{Man}_{5}+3 \mathrm{H}\right]^{3+}\left(n_{\mathrm{H}}>n_{\mathrm{B}}\right)$; $[\mathrm{SRNLTK}+$ $\left.\operatorname{GlcNAC}_{2} \operatorname{Man}_{5}+\mathrm{H}\right]^{2+}\left(n_{\mathrm{H}}=n_{\mathrm{B}}\right)$; and [SRNLTKDR $\left.+\operatorname{GlCNAC}_{2} \operatorname{Man}_{5}+2 \mathrm{H}\right]^{2+}\left(n_{\mathrm{H}}<n_{\mathrm{B}}\right)$. The energy-resolved CID breakdown curves for the remaining glycopeptide ions under study are provided in the Supporting Information (Figures S1-S5). As we have previously noted of other glycopeptide ions [25, 26], the energy-resolved CID behaviors of these precursors were found to be qualitatively similar despite significant differences in proton mobility. In each case, the precursor ions first dissociated to give rise to $Y_{n>1}$ glycan fragments. With further increases in vibrational energy deposition, the $Y_{n>1}$ dissociation products gradually gave way to an abundance of $Y_{1}$ ions. Finally, at sufficiently high collision energies, a decline in the intensity of $Y_{1}$ ions was accompanied by the production of ${ }^{0,2} X_{0}, Y_{0}$, and peptide $b$ and 
$y$ ions. Indeed, for all of the glycopeptide ions studied here, fragment ions of these various types appeared in the same qualitative order with increasing collision energy. Nevertheless, there are substantial quantitative differences in the energy-resolved CID behaviors of the glycopeptide ions. That is, the specific range of $\Delta U$ values which yield a given type of fragment ion is widely varied among precursor ions of different proton mobilities.

To provide a means for quantitative comparison of the vibrational energy required to access glycosidic and peptide backbone cleavages, additional $E_{k, n}$ values were calculated for the corresponding regions of the energy-resolved CID breakdown curves. First, $E_{k, n}$ values which brought about the maximum total intensity of $Y_{n>1}$ glycosidic fragments were determined by fitting the most abundant three to four points of the $\Sigma Y_{n>1}$ curve with a quadratic function (i.e., downward opening parabola), and calculating the inflection point of this function. This was taken to represent the approximate value of $\Delta U$ which provided maximum glycosidic fragmentation. Similarly, the $\Delta U$ value at which the $\Sigma(b+y)$ peptide fragments first constituted $20 \%$ of the total integrated peak area was obtained from a linear fit of the $\Sigma(b+y)$ line in the energy-resolved CID breakdown plot. The $20 \%$ intensity was chosen because, while most of the glycopeptides eventually yielded peptide $b$ and $y$ ions to comprise approximately $40 \%$ or more of the integrated peak area (e.g., Figure $\mathbf{2 b}-\boldsymbol{c}$ ), in two cases only about $20 \%$ of the peak area could be attributed to peptide backbone cleavage products (e.g., Figure 2a). Thus, the $20 \%$ peptide fragment appearance value was used to enable reasonable comparisons among the different precursor ions. In the cases of both maximum $\Sigma Y_{n>1}$ intensity and $20 \% \Sigma(b+y)$ intensity, the $\Delta U$ values of interest were used to calculate the corresponding $E_{k, n}$ values (cf. Equations 1-3). For each glycopeptide ion under study, these $E_{k, n}$ values are summarized in Table 2. Similar to the results for precursor ion survival (cf. Table 1), the $E_{k, n}$ values which yielded maximum $\Sigma Y_{n>1}$ intensity were seen to correlate with the CIR values assigned to each peptide moiety. That is, the $E_{k, n}$ values correlate inversely with proton mobility, as expected. For precursor ions with a readily mobile proton $\left(n_{\mathrm{H}}>n_{\mathrm{B}}\right), E_{k, n}$ values ranged from $4.74-5.56$; for precursor 
ions with a partially mobile proton $\left(n_{\mathrm{H}}=n_{\mathrm{B}}\right), E_{k, n}$ values ranged from $7.67-8.65$; and for the precursor ion with no mobile protons $\left(n_{\mathrm{H}}<n_{\mathrm{B}}\right), E_{k, n}$ value was 10.30 . This underscores the influence of proton mobility upon the vibrational energy necessary to achieve the highest proportion of glycosidic scission products. Contrastingly, the $E_{k, n}$ values that produced peptide $b$ and $y$ ions with an aggregate of $20 \%$ total spectral peak intensity fell in a relatively narrow range. All of these $E_{k, n}$ values ranged from $10.90-13.93$, with no clear relation to the proton mobility of the precursor ion. While initially unexpected, this can be rationalized by noting that, as illustrated by the energy-resolved CID breakdown curves (Figure 2), the $Y_{0}, b$, and $y$ ions appear to be tertiary products of sequential fragmentation, arising largely from further fragmentation of the $Y_{1}$ ion. This suggests that the vibrational energy required to achieve peptide amide bond scission is not a function of the precursor ion proton mobility, but dictated by the proton mobility of the $Y_{1}$ fragment. As these $Y_{1}$ fragments typically had lower charge states than the precursor ion (as will be shown and further discussed below), their proton mobilities were thus lower than those of the corresponding precursor ions. As a consequence, the vibrational degrees of freedom normalized initial precursor ion kinetic energies that bring about polypeptide $b$ and $y$ ions are quite similar among precursor ions - even those with very dissimilar precursor ion survival energies and glycosidic fragment appearance energies - because in each case the peptide sequence ions originate from an intermediate fragment with no mobile protons. These general observations could eventually be of considerable practical usefulness for the deliberate production of amino acid sequence information for unknown glycopeptides by CID.

3.4. CID spectrum comparisons at various proton mobilities. With knowledge of how the precursor ion characteristics of protonated glycopeptides influence their energyresolved CID behaviors, collision energies could be selected such that information on either the oligosaccharide connectivity or the polypeptide sequence was intentionally accessed. Figure 3 provides the CID spectra of the same representative glycopeptide ions for which 
energy-resolved CID breakdown curves were shown in Figure 2. In these spectra, collision energies were chosen to approximate the $E_{k, n}$ values corresponding to maximum $\Sigma Y_{n>1}$ intensity (cf. Table 2). In each case, the CID spectra exhibited extensive series of $Y$-type fragment ions yielding the complete connectivity of the glycan group, down to at least the $Y_{1}$ (and in one case, $Y_{0}$ ) ion. In addition to the $Y$-type dissociation products, a prominent cross-ring cleavage product $\left({ }^{0,2} X_{0}\right)$ was noted in the CID spectrum of the [SRNLTKDR + $\left.\mathrm{GlcNAC}_{2} \mathrm{Man}_{5}+2 \mathrm{H}\right]^{2+}$ ion (Figure $3 \mathrm{c}$ ). While informative as to the general topology and composition of the glycan, none of these spectra were found to yield any detectable peptide backbone fragmentation.

The same glycopeptide ions were then interrogated at collision energies consistent with $E_{k, n}$ values known to bring about $\geq 20 \% \Sigma(b+y)$ intensity (cf. Table 2); the resulting dissociation spectra are provided in Figure 4. Under these conditions, each precursor ion could be fragmented to yield significant information on the amino acid connectivity, with the peptide sequence coverages for the $\left[\mathrm{NLTKDR}+\mathrm{GlCNAC}_{2} \mathrm{Man}_{5}+3 \mathrm{H}\right]^{3+}$, $[\mathrm{SRNLTK}+$ $\left.\mathrm{GlCNAC}_{2} \mathrm{Man}_{5}+2 \mathrm{H}\right]^{2+}$, and $\left[\mathrm{SRNLTKDR}+\mathrm{GlCNAC}_{2} \mathrm{Man}_{5}+2 \mathrm{H}\right]^{2+}$ glycopeptide ions reaching $100.0 \%, 80.0 \%$, and $71.5 \%$, respectively. In addition to peptide sequence ions, $Y_{2}, Y_{1}, Y_{0}$, and ${ }^{0,2} X_{0}$ cleavages were apparent in the spectra. Strikingly, all of the observed peptide sequence ions were singly charged, regardless of the charge state or proton mobility of the initial precursor ions. Moreover, at these collision energies the charge states of all of the $Y_{1}$ ions were such that these fragments lacked a mobile proton (singly charged in the case of the $\left[\right.$ NLTKDR + GICNAc] and [SRNLTK + GICNAC] $Y_{1}$ fragments; doubly charged in the case of the [SRNLTKDR + GICNAc] $Y_{1}$ fragment). The tendency of protonated glycopeptide ions is evidently to either lose charge (likely due to production of carbohydrate oxonium ions [43, 44]) to render a $Y_{1}$ fragment with lower proton mobility than the precursor, or to retain charge in cases where the initial precursor ion had no mobile protons. In all of the cases examined here, this causes the intermediate $Y_{1}$ fragment (which eventually gives rise to peptide $b$ and $y$ ions) to harbor only nonmobile protons. Thus, protonated glycopeptide ions 
can behave quite similarly with respect to the vibrational mode normalized collision energies which bring about peptide backbone fragments, even when they greatly differ in the energetics of precursor ion survival and glycan fragment appearance.

\section{Conclusions}

This report elaborates on the relation of precursor ion proton mobility to the relative energy requirements for glycan and peptide cleavage in vibrational activation / dissociation of representative $\mathrm{N}$-glycopeptides. Both the $50 \%$ precursor ion survival energies and the energies which resulted in optimum production of glycan fragments varied widely among the precursor ions under study, and generally increased with decreasing proton mobility. Conversely, the energies at which peptide backbone fragments were readily accessed exhibited little or no correlation with the proton mobility of the precursor ion. This apparent disconnect is explained by the observation that peptide backbone fragments are products of sequential dissociation, and arise largely from the intermediate $Y_{1}$ fragment. For all of the cases studied here, the $Y_{1}$ fragments were produced in charge states that afforded only nonmobile protons, regardless of the charge state or proton mobility of the precursor ion. An important consequence of this finding is that the degrees of freedom normalized vibrational energy deposition required to render peptide sequence ions was surprisingly similar among the glycopeptide ions studied, even when their other energy-resolved CID characteristics were quite disparate. While further study is needed in order to determine whether these trends are broadly representative of much larger populations of protonated $\mathrm{N}$-glycopeptides, the current results are encouraging in suggesting the potential that glycan cleavage and peptide cleavage can be deliberately accessed for putative unknowns based on characteristics such as charge state and molecular weight. This, in turn, intimates the possibility of an expanded role for CID in N-glycoproteomic analysis. 


\section{Acknowledgements}

This work was supported by the University of Nebraska - Lincoln (including funds from the Nebraska Tobacco Settlement Biomedical Research Development Fund, the CIBC Research Cluster Development Grant, and the Department of Chemistry Faculty / Student Summer Research Program) and the National Science Foundation (Research Experiences for Undergraduates in Chemical Assembly Program at the University of Nebraska - Lincoln, Award CHE 1156560). The authors also acknowledge Yuting Huang and Katherine N. Schumacher for constructive comments on a draft of the manuscript.

\section{Supplementary Material}

The supplementary material includes the remaining energy-resolved CID breakdown curves, as referenced in the text. 


\section{References}

[1] W. Morelle, K. Canis, F. Chirat, V. Faid, J.C. Michalski, The Use of Mass Spectrometry for the Proteomic Analysis of Glycosylation, Proteomics, 6 (2006) 3993-4015.

[2] D.S. Dalpathado, H. Desaire, Glycopeptide Analysis by Mass Spectrometry, The Analyst, 133 (2008) 731-738.

[3] B. Tissot, S.J. North, A. Ceroni, P.-C. Pang, M. Panico, F. Rosati, A. Capone, S.M. Haslam, A. Dell, H.R. Morris, Glycoproteomics: Past, Present and Future, FEBS Lett., 583 (2009) 1728-1735.

[4] S.B. Levery, C. Steentoft, A. Halim, Y. Narimatsu, H. Clausen, S.Y. Vakhrushev, Advances in Mass Spectrometry Driven O-Glycoproteomics, Biochim. Biophys. Acta, 1850 (2015) 33-42.

[5] S.J. North, P.G. Hitchen, S.M. Haslam, A. Dell, Mass Spectrometry in the Analysis of N-Linked and O-Linked Glycans, Curr. Opin. Struct. Biol., 19 (2009) 498-506.

[6] Y. Pasing, A. Sickmann, U. Lewandrowski, N-Glycoproteomics: Mass SpectrometryBased Glycosylation Site Annotation, Biol. Chem., 393 (2012) 249-258.

[7] J.E. Schiel, Glycoprotein Analysis Using Mass Spectrometry: Unraveling the Layers of Complexity, Anal. Bioanal. Chem., 404 (2012) 1141-1149.

[8] V. Kolli, K.N. Schumacher, E.D. Dodds, Engaging Challenges in Glycoproteomics: Recent Advances in MS-Based Glycopeptide Analysis, Bioanalysis, 7 (2015) 113131.

[9] W.R. Alley, B.F. Mann, M.V. Novotny, High-Sensitivity Analytical Approaches for the Structural Characterization of Glycoproteins, Chem. Rev., 113 (2013) 2668-2732.

[10] H. Desaire, Glycopeptide Analysis, Recent Developments and Applications, Mol. Cell. Proteomics, 12 (2013) 893-901. 
[11] Y. Huang, A.S. Gelb, E.D. Dodds, Carbohydrate and Glycoconjugate Analysis by Ion Mobility Mass Spectrometry: Opportunities and Challenges, Curr. Metabolomics, 1 (2013) 291-305.

[12] M. Thaysen-Andersen, N.H. Packer, Advances in LC-MS/MS-Based Glycoproteomics: Getting Closer to System-Wide Site-Specific Mapping of the $\mathrm{N}$ - and $\mathrm{O}-$ Glycoproteome, Biochim. Biophys. Acta, 1844 (2014) 1437-1452.

[13] M. Wuhrer, M.I. Catalina, A.M. Deelder, C.H. Hokke, Glycoproteomics based on Tandem Mass Spectrometry of Glycopeptides, J. Chromatogr. B, 849 (2007) 115128.

[14] E.D. Dodds, Gas-Phase Dissociation of Glycosylated Peptide Ions, Mass Spectrom. Rev., 31 (2012) 666-682.

[15] H.J. An, J.W. Froehlich, C.B. Lebrilla, Determination of Glycosylation Sites and SiteSpecific Heterogeneity in Glycoproteins, Curr. Opin. Chem. Biol., 13 (2009) 421 426.

[16] J. Nilsson, A. Halim, A. Grahn, G. Larson, Targeting the Glycoproteome, Glycoconj. J., 30 (2013) 119-136.

[17] L. Zhang, J.P. Reilly, Extracting Both Peptide Sequence and Glycan Structural Information by $157 \mathrm{~nm}$ Photodissociation of N-Linked Glycopeptides, J. Proteome Res., 8 (2008) 734-742.

[18] J.A. Madsen, B.J. Ko, H. Xu, J.A. Iwashkiw, S.A. Robotham, J.B. Shaw, M.F. Feldman, J.S. Brodbelt, Concurrent Automated Sequencing of the Glycan and Peptide Portions of O-Linked Glycopeptide Anions by Ultraviolet Photodissociation Mass Spectrometry, Anal. Chem., 85 (2013) 9253-9261.

[19] J.T. Adamson, K. Hakansson, Infrared Multiphoton Dissociation and Electron Capture Dissociation of High-Mannose Type Glycopeptides, J. Proteome Res., 5 (2006) 493501. 
[20] L. Bindila, K. Steiner, C. Schaffer, P. Messner, M. Mormann, J. Peter-Katalinic, Sequencing of O-Glycopeptides Derived from an S-Layer Glycoprotein of Geobacillus Stearothermophilus NRS 2004/3a Containing up to 51 Monosaccharide Residues at a Single Glycosylation Site by Fourier Transform Ion Cyclotron Resonance Infrared Multiphoton Dissociation Mass Spectrometry, Anal. Chem., 79 (2007) 3271-3279.

[21] R.R. Seipert, E.D. Dodds, B.H. Clowers, S.M. Beecroft, J.B. German, C.B. Lebrilla, Factors That Influence Fragmentation Behavior of N-Linked Glycopeptide Ions, Anal. Chem., 80 (2008) 3684-3692.

[22] R.R. Seipert, E.D. Dodds, C.B. Lebrilla, Exploiting Differential Dissociation Chemistries of O-Linked Glycopeptide Ions for the Localization of Mucin-Type Protein Glycosylation, J. Proteome Res., 8 (2009) 493-501.

[23] Z.M. Segu, Y. Mechref, Characterizing Protein Glycosylation Sites through HigherEnergy C-Trap Dissociation, Rapid Commun. Mass Spectrom., 24 (2010) 12171225.

[24] Q. Cao, X. Zhao, Q. Zhao, X. Lv, C. Ma, X. Li, Y. Zhao, B. Peng, W. Ying, X. Qian, Strategy Integrating Stepped Fragmentation and Glycan Diagnostic Ion-Based Spectrum Refinement for the Identification of Core Fucosylated Glycoproteome using Mass Spectrometry, Anal. Chem., 86 (2014) 6804-6811.

[25] V. Kolli, E.D. Dodds, Energy-Resolved Collision-Induced Dissociation Pathways of Model N-Linked Glycopeptides: Implications for Capturing Glycan Connectivity and Peptide Sequence in a Single Experiment, The Analyst, 139 (2014) 2144-2153.

[26] F. Aboufazeli, V. Kolli, E.D. Dodds, A Comparison of Energy-Resolved Vibrational Activation / Dissociation Characteristics of Protonated and Sodiated High Mannose N-Glycopeptides, J. Am. Soc. Mass Spectrom., 26 (2015) 587-595.

[27] M. Tajiri, M. Kadoya, Y. Wada, Dissociation Profile of Protonated Fucosyl Glycopeptides and Quantitation of Fucosylation Levels of Glycoproteins by Mass Spectrometry, J. Proteome Res., 8 (2009) 688-693. 
[28] K. Vekey, O. Ozohanics, E. Toth, A. Jeko, A. Revesz, J. Krenyacz, L. Drahos, Fragmentation Characteristics of Glycopeptides, Int. J. Mass Spectrom., 345-347 (2013) 71-79.

[29] A.R. Dongré, J.L. Jones, Á. Somogyi, V.H. Wysocki, Influence of Peptide Composition, Gas-Phase Basicity, and Chemical Modification on Fragmentation Efficiency: Evidence for the Mobile Proton Model, J. Am. Chem. Soc., 118 (1996) 8365-8374.

[30] V.H. Wysocki, G. Tsaprailis, L.L. Smith, L.A. Breci, Mobile and Localized Protons: A Framework for Understanding Peptide Dissociation, J. Mass Spectrom., 35 (2000) 1399-1406.

[31] A. Varki, R.D. Cummings, J.D. Esko, H.H. Freeze, P. Stanley, J.D. Marth, C.R. Bertozzi, G.W. Hart, M.E. Etzler, Symbol Nomenclature for Glycan Representation, Proteomics, 9 (2009) 5398-5399.

[32] P. Roepstorff, J. Fohlman, Proposal for a Common Nomenclature for Sequence Ions in Mass Spectra of Peptides Biomed. Mass Spectrom., 11 (1984) 601.

[33] B. Domon, C.E. Costello, A Systematic Nomenclature for Carbohydrate Fragmentations in FAB-MS/MS Spectra of Glycoconjugates, Glycoconj. J., 5 (1988) 397-409.

[34] P. Juhasz, S.A. Martin, The Utility of Nonspecific Proteases in the Characterization of Glycoproteins by High-Resolution Time-of-Flight Mass Spectrometry, Int. J. Mass Spectrom. Ion Proc., 169/170 (1997) 217-230.

[35] H.J. An, T.R. Peavy, J.L. Hedrick, C.B. Lebrilla, Determination of N-Glycosylation Sites and Site Heterogeneity in Glycoproteins, Anal. Chem., 75 (2003) 5628-5637.

[36] B.H. Clowers, E.D. Dodds, R.R. Seipert, C.B. Lebrilla, Site Determination of Protein Glycosylation based on Digestion with Immobilized Nonspecific Proteases and Fourier Transform Ion Cyclotron Resonance Mass Spectrometry, J. Proteome Res., 6 (2007) 4032-4040. 
[37] E.D. Dodds, R.R. Seipert, B.H. Clowers, J.B. German, C.B. Lebrilla, Analytical Performance of Immobilized Pronase for Glycopeptide Footprinting and Implications for Surpassing Reductionist Glycoproteomics, J. Proteome Res., 8 (2009) 502-512.

[38] A. Memboeuf, A. Nasioudis, S. Indelicato, F. Pollreisz, A. Kuki, S. Keki, O.F. van den Brink, K. Vekey, L. Drahos, Size Effect on Fragmentation in Tandem Mass Spectrometry, Anal. Chem., 82 (2010) 2294-2302.

[39] S. Indelicato, D. Bongiorno, S. Indelicato, L. Drahos, V. Turco Liveri, L. Turiák, K. Vékey, L. Ceraulo, Degrees of Freedom Effect on Fragmentation in Tandem Mass Spectrometry of Singly Charged Supramolecular Aggregates of Sodium Sulfonates, J. Mass Spectrom., 48 (2013) 379-383.

[40] E.A. Kapp, F. Schutz, G.E. Reid, J.S. Eddes, R.L. Moritz, R.A. O'Hair, T.P. Speed, R.J. Simpson, Mining a Tandem Mass Spectrometry Database to Determine the Trends and Global Factors Influencing Peptide Fragmentation, Anal. Chem., 75 (2003) 6251-6264.

[41] A.C. Gucinski, E.D. Dodds, W.Z. Li, V.H. Wysocki, Understanding and Exploiting Peptide Fragment Ion Intensities Using Experimental and Informatic Approaches, Meth. Mol. Biol., 604 (2010) 73-94.

[42] B. Paizs, S. Suhai, Fragmentation Pathways of Protonated Peptides, Mass Spectrom. Rev., 24 (2005) 508-548.

[43] G. Hart-Smith, M.J. Raftery, Detection and Characterization of Low Abundance Glycopeptides via Higher-Energy C-Trap Dissociation and Orbitrap Mass Analysis, J. Am. Soc. Mass Spectrom., 23 (2012) 124-140.

[44] J.W. Froehlich, E.D. Dodds, M. Wilhelm, O. Serang, J.A. Steen, R.S. Lee, A Classifier Based on Accurate Mass Measurements to Aid Large Scale, Unbiased Glycoproteomics, Mol. Cell. Proteomics, 12 (2013) 1017-1025. 


\section{Figure Captions}

Figure 1. Precursor ion survival curves for each of the glycopeptide ions studied. The sequences of the peptide moieties and overall ion charge states are given in the inset. In addition, each glycopeptide harbored the $\mathrm{GlcNAc}_{2} \mathrm{Man}_{5} \mathrm{~N}$-glycan. Amino acid residues with basic side chains are shown in bold, while the glycosylated asparagine residue is underlined. Each data point represents the mean of three replicate measurements; error bars, where visible, represent the standard deviation.

Figure 2. Energy-resolved CID breakdown curves for the $\left[\mathrm{NLTKDR}+\mathrm{GlCNAc}_{2} \mathrm{Man}_{5}+3 \mathrm{H}\right]^{3+}$ $\left(\boldsymbol{a} ; n_{\mathrm{H}}>n_{\mathrm{B}}\right),\left[\mathrm{SRNLTK}+\mathrm{GlCNAC}_{2} \mathrm{Man}_{5}+2 \mathrm{H}\right]^{2+}\left(\boldsymbol{b} ; n_{\mathrm{H}}=n_{\mathrm{B}}\right)$, and [SRNLTKDR + $\left.\mathrm{GlcNAC}_{2} \mathrm{Man}_{5}+2 \mathrm{H}\right]^{2+}\left(\boldsymbol{c} ; n_{\mathrm{H}}<n_{\mathrm{B}}\right)$ glycopeptide ions. Additional explanation is provided in the caption to Figure 1.

Figure 3. CID spectra for the $\left[N L T K D R+\operatorname{GlCNAC}_{2} \mathrm{Man}_{5}+3 \mathrm{H}\right]^{3+}\left(\boldsymbol{a} ; n_{\mathrm{H}}>n_{\mathrm{B}}\right)$, $[\mathrm{SRNLTK}+$ $\left.\operatorname{GlcNAC}_{2} \operatorname{Man}_{5}+2 \mathrm{H}\right]^{2+}\left(\boldsymbol{b} ; n_{\mathrm{H}}=n_{\mathrm{B}}\right)$, and $\left[\mathrm{SRNLTKDR}+\operatorname{GlcNAc}_{2} \operatorname{Man}_{5}+2 \mathrm{H}\right]^{2+}\left(\boldsymbol{c} ; n_{\mathrm{H}}<\right.$ $\left.n_{\mathrm{B}}\right)$. The applied precursor acceleration potentials $(\Delta U)$ are indicated, and were chosen to bring about the maximum proportion of glycosidic bond cleavage products (cf. Table 2). Cleavage maps summarizing the fragmentation of each glycopeptide are provided to the right of each spectrum.

Figure 4. CID spectra for the $\left[\mathrm{NLTKDR}+\mathrm{GlCNAC}_{2} \mathrm{Man}_{5}+3 \mathrm{H}\right]^{3+}\left(\boldsymbol{a} ; n_{\mathrm{H}}>n_{\mathrm{B}}\right)$, $[\mathrm{SRNLTK}+$ $\left.\operatorname{GlcNAC}_{2} \operatorname{Man}_{5}+2 \mathrm{H}\right]^{2+}\left(\boldsymbol{b} ; n_{\mathrm{H}}=n_{\mathrm{B}}\right)$, and $\left[\mathrm{SRNLTKDR}+\operatorname{GlcNAc}_{2} \operatorname{Man}_{5}+2 \mathrm{H}\right]^{2+}\left(\boldsymbol{c} ; n_{\mathrm{H}}<\right.$ $\left.n_{\mathrm{B}}\right)$. The applied precursor acceleration potentials $(\Delta U)$ are indicated, and were chosen to achieve peptide backbone cleavage such that these products constituted approximately $20 \%$ of the integrated peak area (cf. Table 2). Cleavage maps summarizing the fragmentation of each glycopeptide are provided to the right of each spectrum. 


\section{Figures}

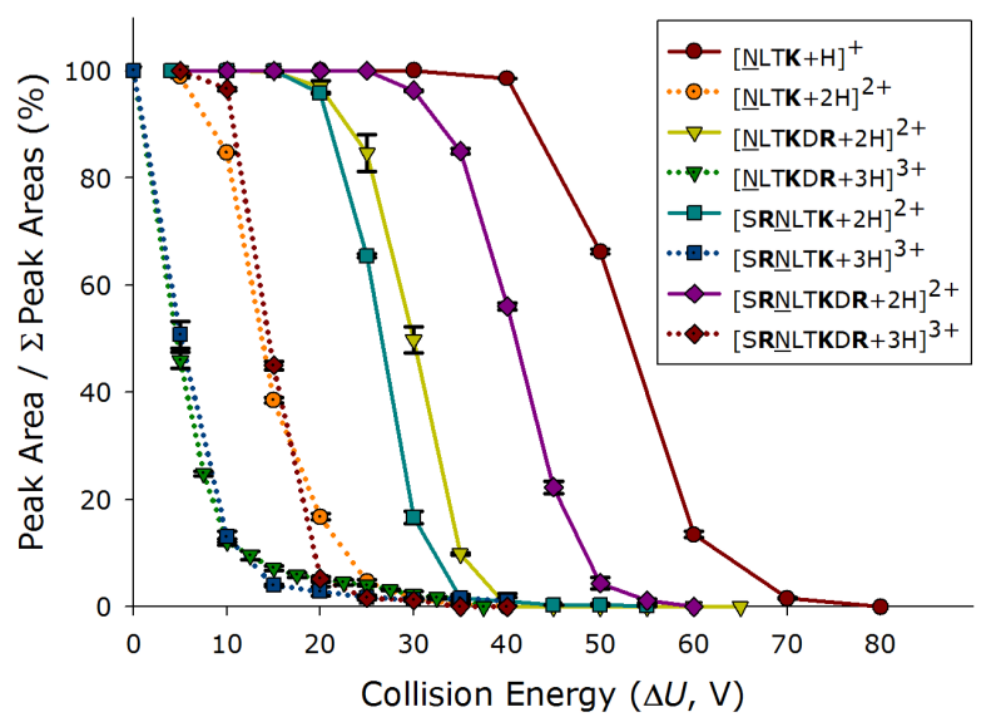

Figure 1. 

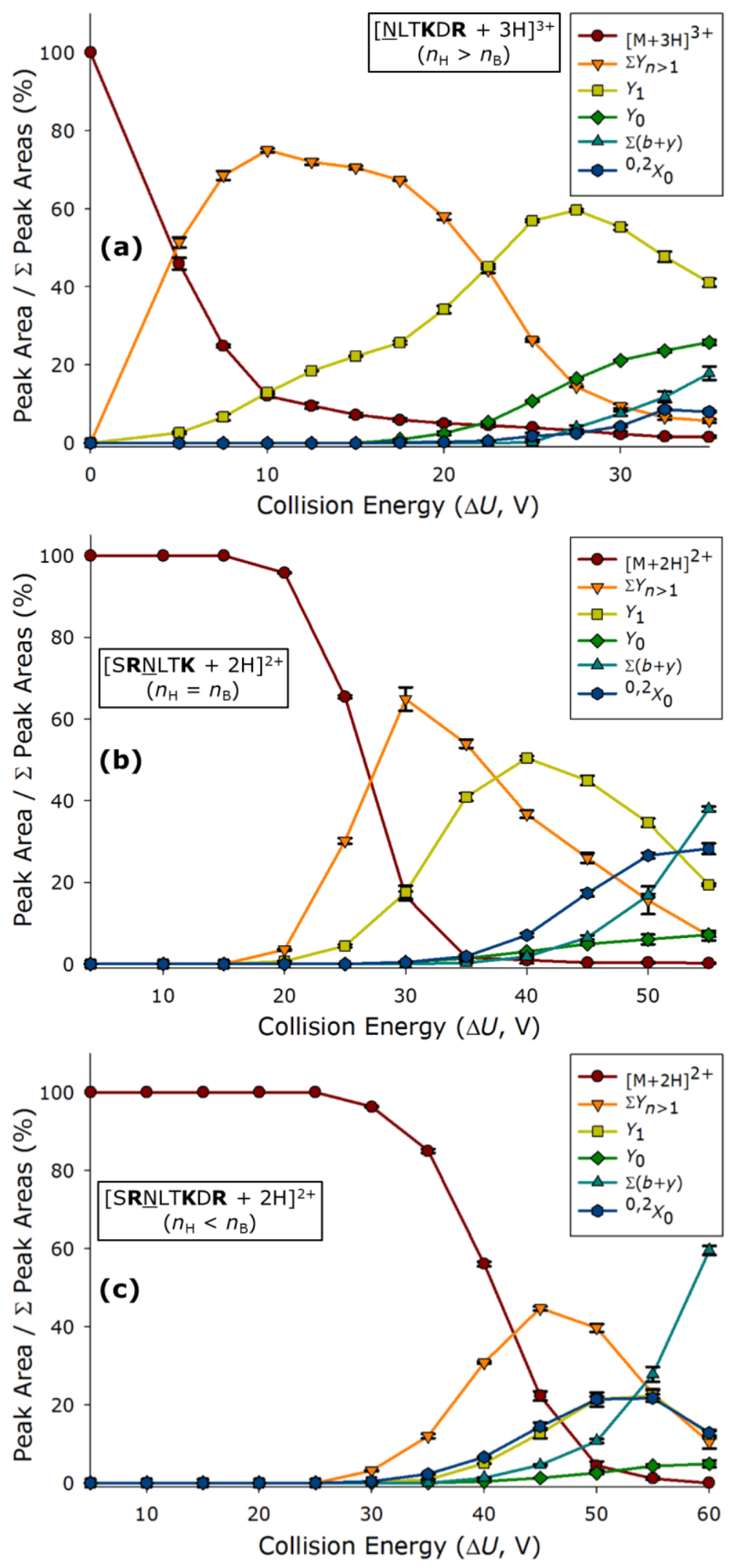

Figure 2. 

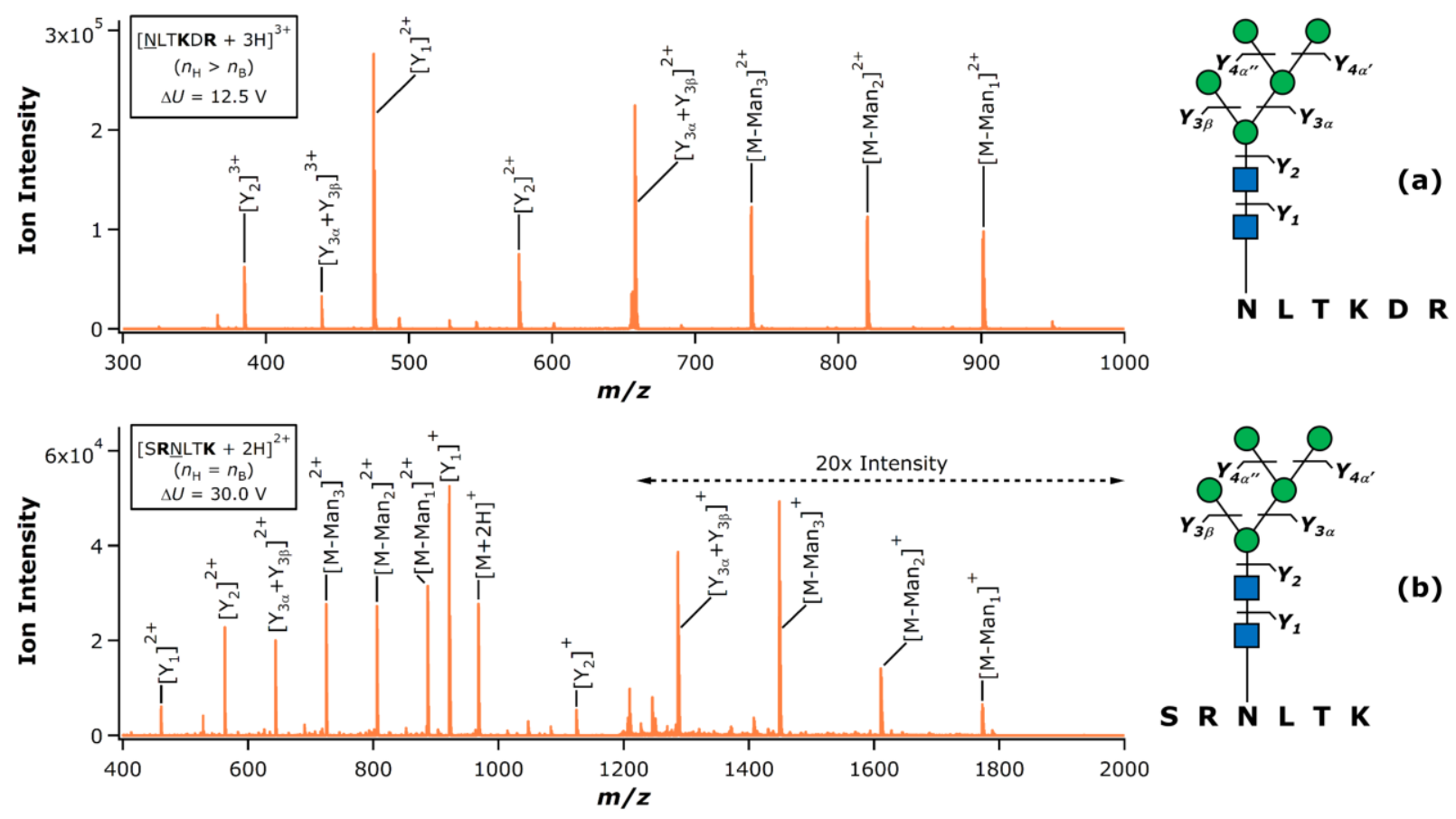

(b)

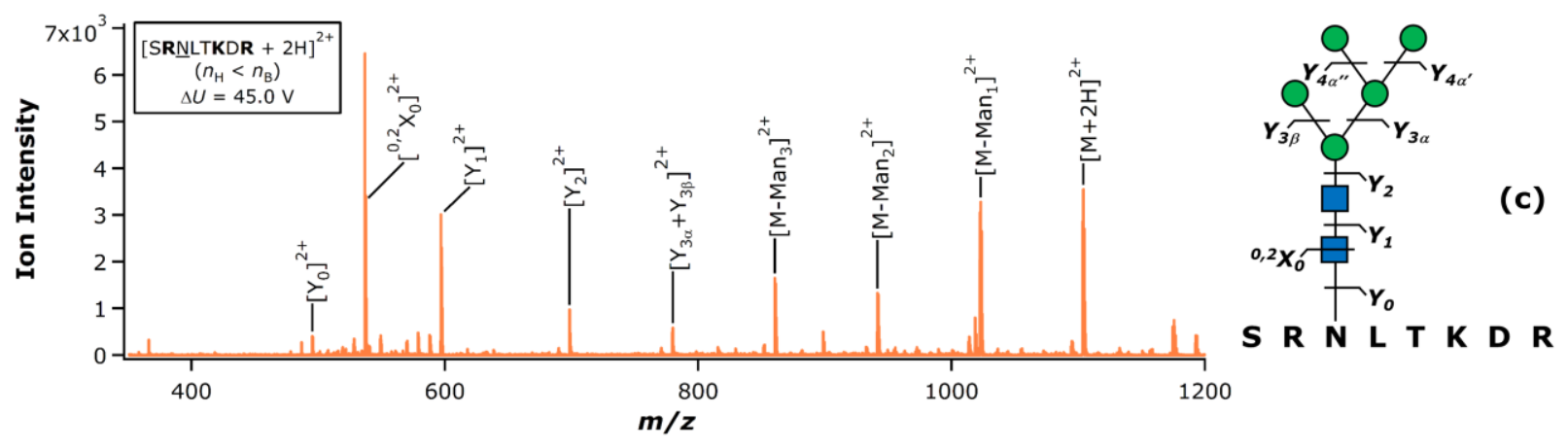

Figure 3. 

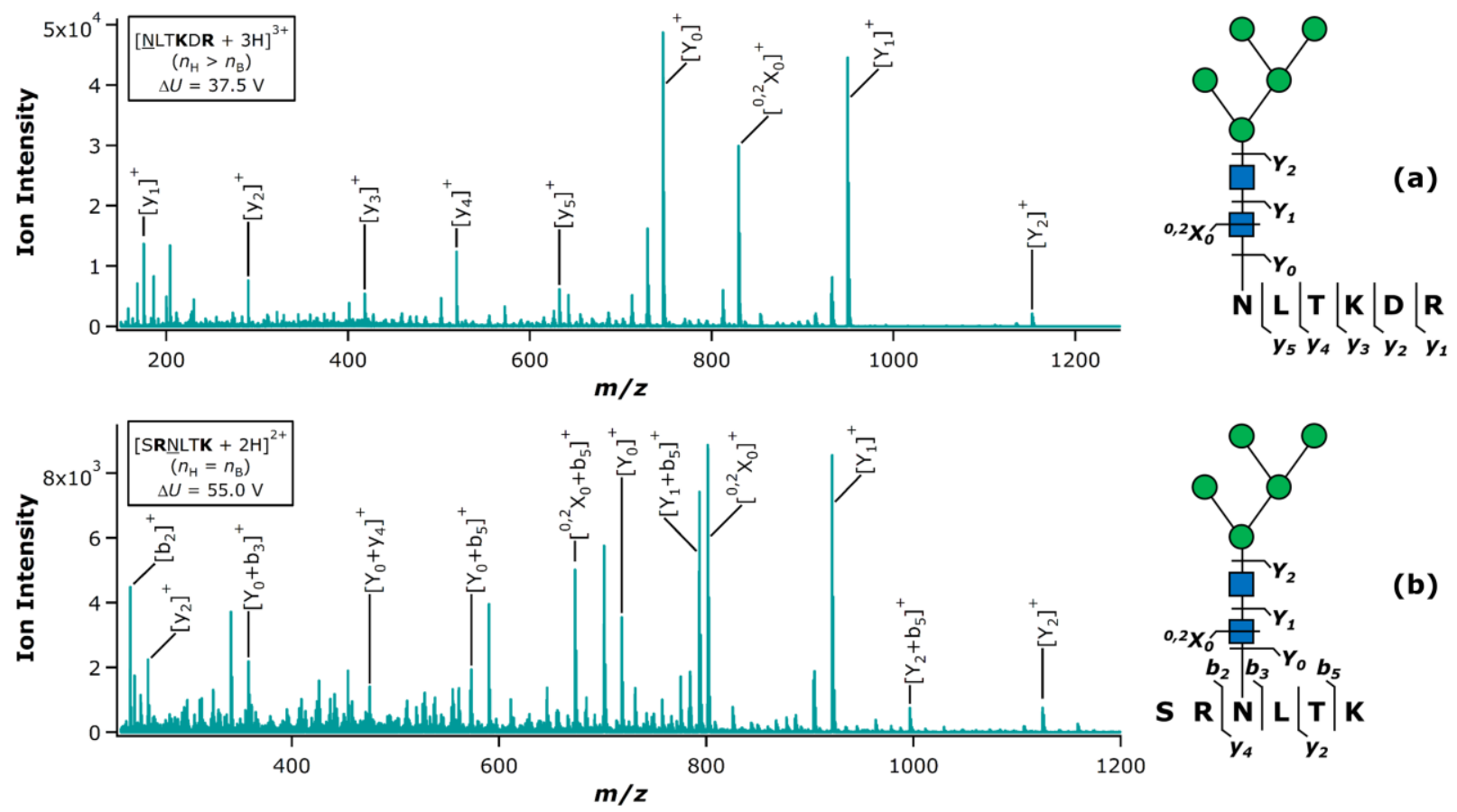

(b)
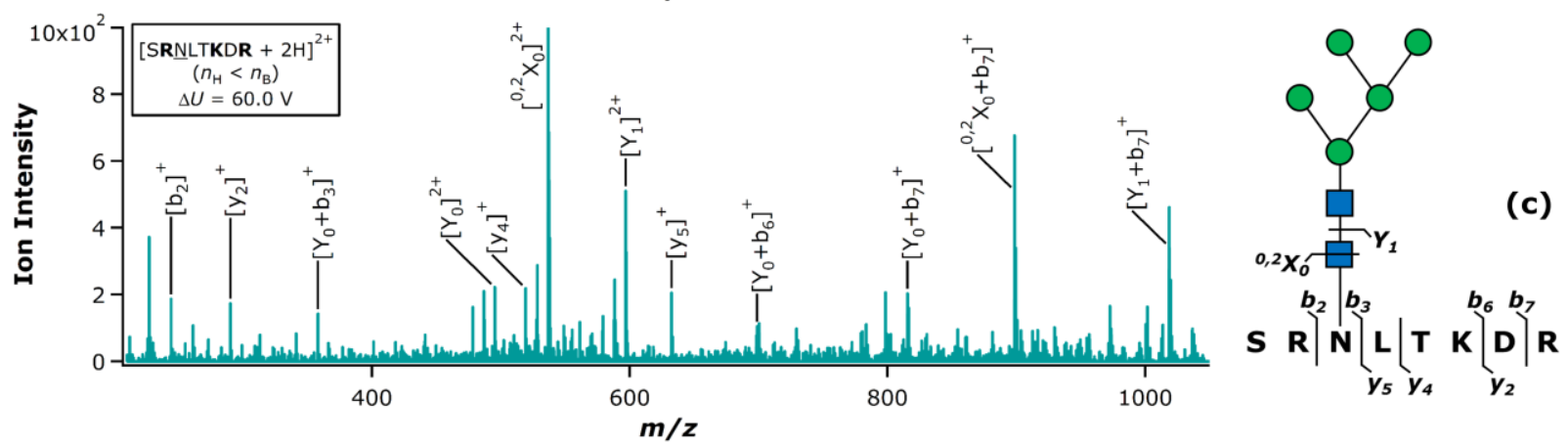

Figure 4. 


\section{Table Captions}

Table 1. Potential differences $(\Delta U)$ and degrees of freedom normalized initial precursor ion kinetic energies $\left(E_{k, n}\right)$ resulting in $50 \%$ precursor ion survival of each glycopeptide ion studied. The number of charge-carrying protons $\left(n_{H}\right)$ is indicated relative to the number of basic amino acid residues $\left(n_{\mathrm{B}}\right)$, and precursor ion charge sates $(z)$ and vibrational degrees of freedom $\left(f_{v}\right)$ are given. Within the glycopeptide compositions, basic amino acid residues are bolded, and the glycosylated asparagine residue is underlined. The "cleavage intensity ratio" (CIR) is also provided for each precursor ion according to Kapp et al [40]. Larger CIR values indicate lower proton mobility, as estimated based upon the precursor ion charge state and amino acid composition.

Table 2. Potential differences $(\Delta U)$ and degrees of freedom normalized initial precursor ion kinetic energies $\left(E_{k, n}\right)$ resulting in the maximum proportion of glycosidic bond cleavage products $\left(\sum Y_{n>1}\right)$, and those necessary for peptide backbone cleavage products $(\Sigma b, y)$ to constitute $20 \%$ of the integrated peak area for each glycopeptide ion. Additional explanation is provided in the caption to Table 1. 


\section{Tables}

Table 1.

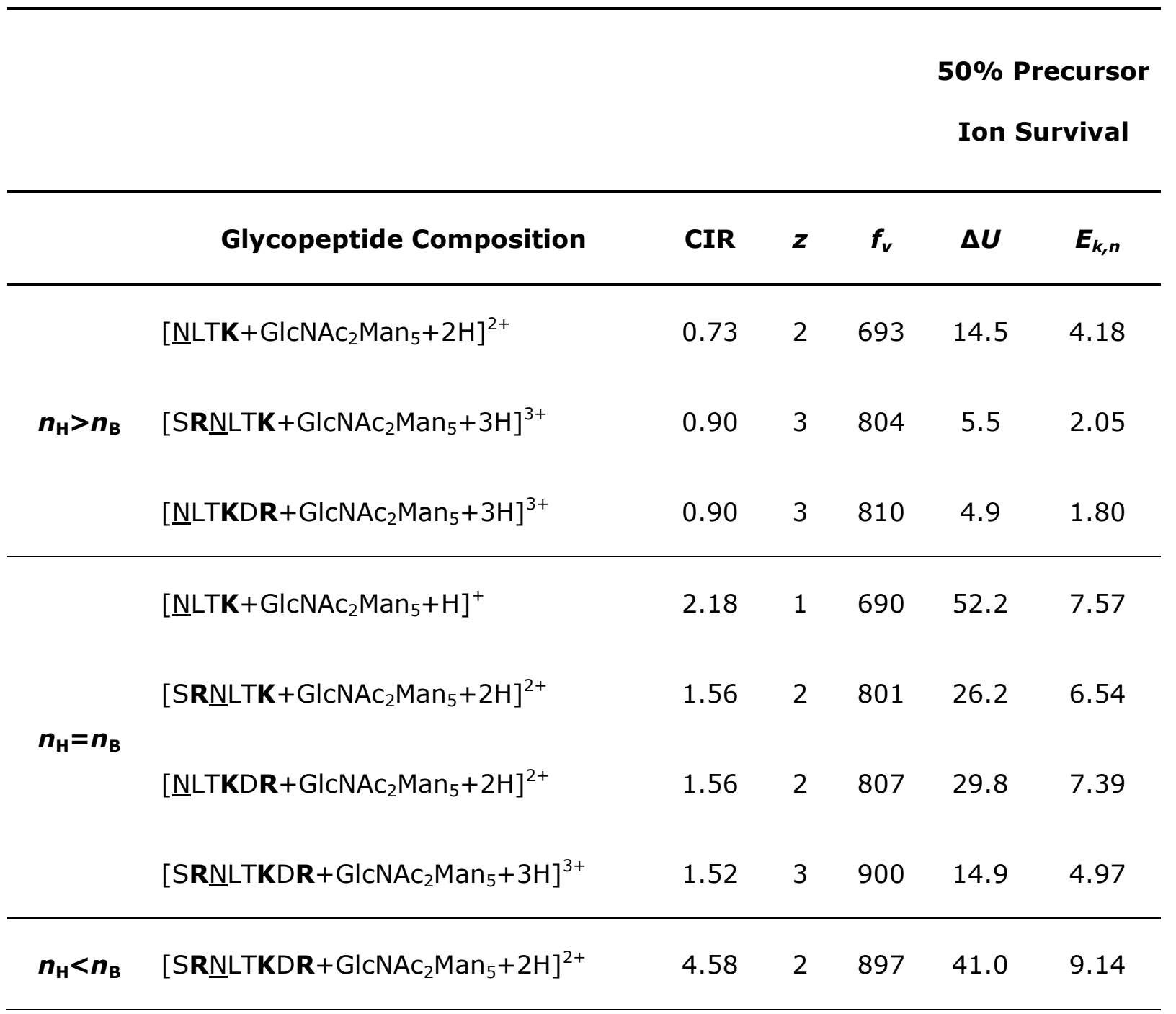


Table 2.

\begin{tabular}{|c|c|c|c|c|c|c|c|c|}
\hline & & & & & \multicolumn{2}{|c|}{$\begin{array}{l}\text { Max } \sum Y_{n>1} \\
\text { Intensity }\end{array}$} & \multicolumn{2}{|c|}{$\begin{array}{c}20 \% \Sigma(b, y) \\
\text { Intensity }\end{array}$} \\
\hline & Glycopeptide Composition & CIR & $z$ & $f_{v}$ & $\Delta \boldsymbol{U}$ & $E_{k, n}$ & $\Delta \boldsymbol{U}$ & $E_{k, n}$ \\
\hline \multirow{3}{*}{$n_{H}>n_{B}$} & {$\left[\underline{\mathrm{NLTK}}+\mathrm{GlcNAc}_{2} \mathrm{Man}_{5}+2 \mathrm{H}\right]^{2+}$} & 0.73 & 2 & 693 & 17.0 & 4.91 & 42.9 & 12.38 \\
\hline & {$\left[\mathrm{SR} \underline{N} L T K+\mathrm{GlcNAC}_{2} \mathrm{Man}_{5}+3 \mathrm{H}\right]^{3+}$} & 0.90 & 3 & 804 & 14.9 & 5.56 & 35.3 & 13.17 \\
\hline & {$\left[\underline{\mathrm{NLTKDR}}+\mathrm{GlcNAc}_{2} \mathrm{Man}_{5}+3 \mathrm{H}\right]^{3+}$} & 0.90 & 3 & 810 & 12.8 & 4.74 & 35.2 & 13.04 \\
\hline \multirow{5}{*}{$n_{H}=n_{B}$} & {$\left[\underline{\mathrm{NLTK}}+\mathrm{GlCNAc}_{2} \mathrm{Man}_{5}+\mathrm{H}\right]^{+}$} & 2.18 & 1 & 690 & 58.8 & 8.52 & 75.2 & 10.90 \\
\hline & {$\left[\mathrm{SR} \underline{\mathrm{NLTK}}+\mathrm{GlcNAc}_{2} \mathrm{Man}_{5}+2 \mathrm{H}\right]^{2+}$} & 1.56 & 2 & 801 & 31.3 & 7.82 & 49.9 & 12.46 \\
\hline & & & & & & & & \\
\hline & {$\left[\underline{\mathrm{NLTKDR}}+\mathrm{GlcNAc}_{2} \mathrm{Man}_{5}+2 \mathrm{H}\right]^{2+}$} & 1.56 & 2 & 807 & 34.9 & 8.65 & 56.2 & 13.93 \\
\hline & {$\left[\mathrm{SR} \underline{\mathrm{N} L T K D R}+\mathrm{GlcNAC}_{2} \mathrm{Man}_{5}+3 \mathrm{H}\right]^{3+}$} & 1.52 & 3 & 900 & 23.0 & 7.67 & 34.5 & 11.50 \\
\hline$n_{H}<n_{B}$ & {$\left[\mathrm{SR} \underline{N} L T K D R+\mathrm{GlcNAC}_{2} \mathrm{Man}_{5}+2 \mathrm{H}\right]^{2+}$} & 4.58 & 2 & 897 & 46.2 & 10.30 & 52.4 & 11.68 \\
\hline
\end{tabular}




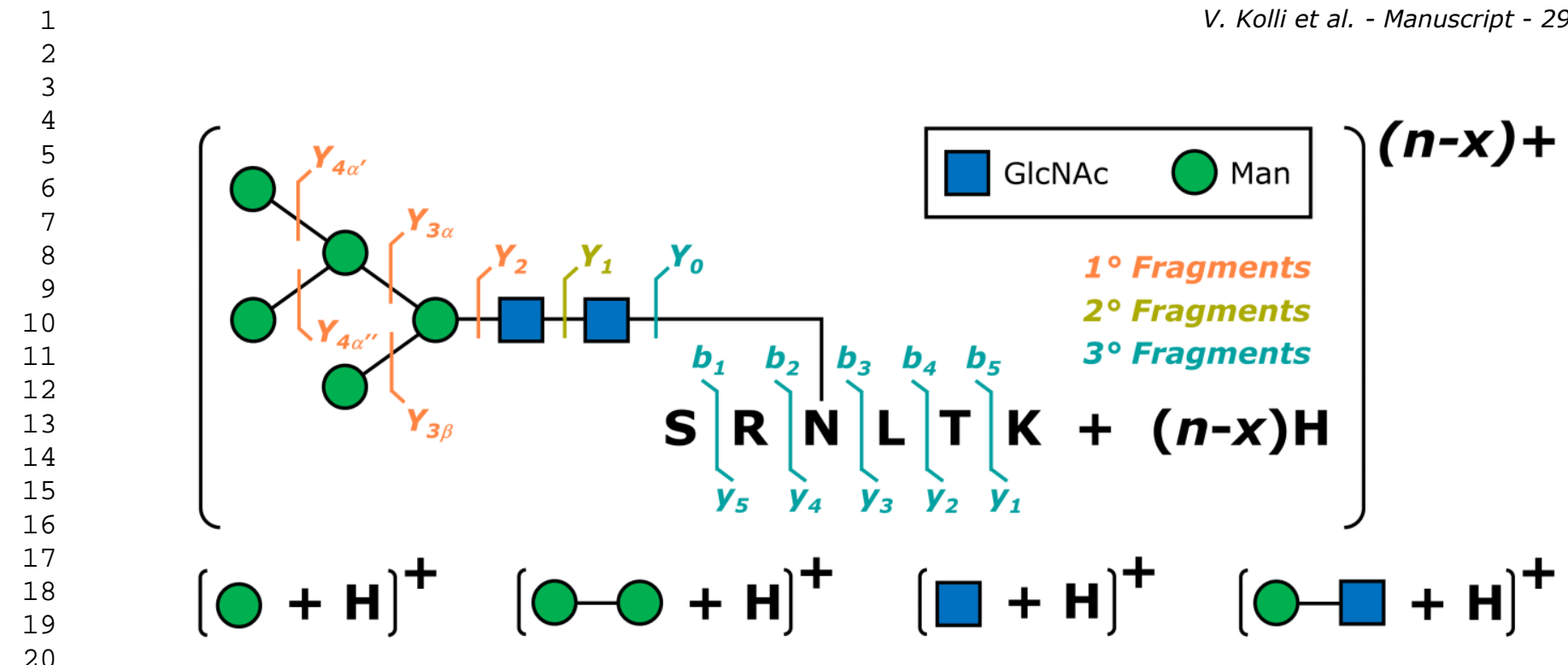

Graphical abstract. 